\title{
Mutations in the Arabidopsis homoserine kinase gene DMR1 confer enhanced resistance to Fusarium culmorum and F. graminearum
}

\author{
Helen C Brewer, Nathaniel D Hawkins and Kim E Hammond-Kosack
}

\begin{abstract}
Background: Mutation of Arabidopsis DMR1, encoding homoserine kinase, leads to elevation in homoserine and foliar resistance to the biotrophic pathogens Hyaloperonospora arabidopsidis and Oidium neolycopersici through activation of an unidentified defence mechanism. This study investigates the effect of mutation of dmrl on resistance to the ascomycete pathogens Fusarium graminearum and F. culmorum, which cause Fusarium Ear Blight (FEB) disease on small grain cereals.

Results: We initially found that the dmr1-2 mutant allele confers increased resistance to $F$. culmorum and $F$. graminearum silique infection, and decreased colonisation of rosette leaves. Meanwhile the dmr1-1 allele supports less rosette leaf colonisation but has wild type silique resistance. Three additional $d m r 1$ alleles were subsequently examined for altered F. culmorum susceptibility and all showed increased silique resistance, while leaf colonisation was reduced in two (dmr1-3 and dmr1-4). Amino acid analysis of dmr1 siliques revealed homoserine accumulation, which is undetectable in wild type plants. Exogenous application of L-homoserine reduced bud infection in both $d m r 1$ and wild type plants, whilst D-homoserine application did not. Delayed leaf senescence was also observed in dmr1 plants compared to wild type and correlated with reduced Fusarium leaf colonisation.
\end{abstract}

Conclusions: These findings suggest that common Arabidopsis DMR1 mediated susceptibility mechanisms occur during infection by both obligate biotrophic oomycete and hemi-biotrophic fungal pathogens, not only in vegetative but also in reproductive plant tissues. This has the potential to aid the development of cereal crops with enhanced resistance to FEB.

Keywords: Arabidopsis, Fusarium culmorum, Fusarium graminearum, Homoserine kinase, Disease resistance, Gain of function, Fusarium head scab

\section{Background}

Fusarium Ear Blight (FEB) disease, also known as Fusarium head scab disease, is a globally significant threat to the floral tissues of small grain cereal crops such as wheat, barley and maize, caused by several species of ascomycete fungi of the genus Fusarium. The main causal agents of FEB in the UK are Fusarium graminearum and $F$. culmorum (Dean et al. [1], Goswami and Kistler [2], Parry et al. [3]). The disease can cause huge crop losses in epidemic years due to reduction in grain yield or grain quality and via the contamination of the grain

\footnotetext{
* Correspondence: kim.hammond-kosack@rothamsted.ac.uk Department of Plant Biology and Crop Science, Rothamsted Research,
} Harpenden AL5 2JQ, UK

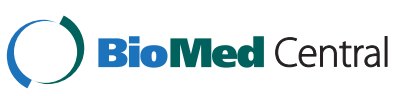

(c) 2014 Brewer et al.; licensee BioMed Central Ltd. This is an Open Access article distributed under the terms of the Creative Commons Attribution License (http://creativecommons.org/licenses/by/4.0), which permits unrestricted use, distribution, and reproduction in any medium, provided the original work is properly credited. The Creative Commons Public Domain Dedication waiver (http://creativecommons.org/publicdomain/zero/1.0/) applies to the data made available in this article unless otherwise stated. with mycotoxins such as deoxynivalenol (DON) which make grain unsafe for human and animal consumption (Rocha et al. [4]). No commercially available wheat cultivars are fully resistant to FEB and the basis of resistance is complex and major quantitative trait loci (QTL) based (Bai and Shaner [5], Buerstmayr et al. [6], Jayatilake et al. [7], Zhou et al. [8]).

Both F. graminearum and F. culmorum infect the floral and silique tissue of Arabidopsis thaliana under experimental conditions, thereby providing a tractable model for the study of host pathogen interactions during FEB disease (Urban et al. [9]). A role for a number of Arabidopsis genes in resistance/susceptibility to Fusarium has been identified using this pathosystem (Cuzick et al. [10], Cuzick et al. [11], Makandar et al. [12], Makandar et al.

ras otherwise stated. 
[13], Savitch et al. [14], Van Hemelrijck et al. [15]). In addition, transgenic and chemical approaches have been used to alter Arabidopsis leaf and floral susceptibility to FEB causing Fusarium species (Asano et al. [16], Ferrari et al. [17], Kaur et al. [18], Koch et al. [19], Schreiber et al. [20]). These findings have the potential to improve defence against FEB in cereal crops using conventional and transgenic approaches, and also enhance our understanding of defence responses in plant reproductive tissues.

The Arabidopsis downy mildew resistant (dmr) mutants were isolated from a gain of function screen for resistance to the oomycete pathogen Hyaloperonospora arabidopsidis, following ethyl methanesulfonate (EMS) mutagenesis of plants of the susceptible genotype Ler-0 harbouring the enhanced disease susceptibility mutation eds1-2 (Van Damme et al. [21]). The eds1-2 mutation in Ler-0 has previously been shown not to alter the interaction outcome between F. culmorum and Arabidopsis floral or silique tissue (Cuzick et al. [11]). Of the five $d m r$ mutant alleles identified, three $(d m r 3, d m r 4, d m r 5)$ showed constitutive expression of the salicylic acid mediated defence related gene $P R-1$. The remaining mutants, $d m r 1$ and $d m r 6$, were mapped and identified as encoding mutations in the Arabidopsis homoserine kinase, and a putative 2-oxoglurarate oxygenase, respectively (van Damme et al. [22], van Damme et al. [23]). DMR6 is associated with salicylic acid mediated defence signalling but is required for $H$. arabidopsidis susceptibility. Mutation of $d m r 1$ results in accumulation of homoserine in non-inoculated plants, and exogenous application of L-homoserine co-incident with $H$. arabidopsidis inoculation confers resistance in wild type plants. However, the precise role of L-homoserine in resistance is not known.

We used the Fusarium - Arabidopsis floral pathosystem (Urban et al. [9]) to assess the effects of the dmr mutations on Fusarium susceptibility in Arabidopsis floral, silique and rosette leaf tissue. Mutants dmr1-1, dmr1-2, $d m r 5$ and $d m r 6$ (all of which also carry the eds1-2 mutation) were initially investigated; $d m r 3$ and $d m r 4$ have pleiotropic dwarf phenotypes which affect floral morphology and were therefore unsuitable for inclusion in this study. Here we present the novel finding that reduced function of the Arabidopsis homoserine kinase DMR1 confers resistance to F. graminearum and F. culmorum in siliques and/or reduces colonisation of rosette leaf tissues, with varying levels of resistance conferred by different $d m r 1$ mutant alleles. The siliques of $d m r 1$ plants accumulate homoserine but are not depleted in amino acids such as threonine and methionine which are downstream products of homoserine kinase activity. We also find that mutation of DMR1 results in delayed leaf senescence which may relate to the observed reduced leaf colonisation phenotype. Exogenous application of L-homoserine reduces floral and silique disease severity in both eds1-2 and $d m r 1$ plants, but does not inhibit in vitro Fusarium growth.

\section{Results}

A selection of the Arabidopsis downy mildew resistant mutants have altered susceptibility to Fusarium culmorum silique infection and rosette leaf colonisation

The Arabidopsis mutants dmr1-1,dmr1-2, dmr5 and dmr6, which were generated in the Ler-0 eds1-2 background, were screened for altered susceptibility to F. culmorum infection compared to eds1-2. Wild type Ler-0 was also included in the assay. Following spray inoculation with F. culmorum spores, the plants were scored for floral and silique disease levels, along with rosette leaf infection and number of uninfected green siliques, after 7, 11 and 14 days (Figures 1 and 2). There was no statistically significant difference in floral FAD (Fusarium-Arabidopsis Disease) score (Urban et al. [9]) between the genotypes tested $\left(\mathrm{F}_{4,93}=0.7, \mathrm{p}=0.591\right)$ at any of the time points assessed, with disease progressing at an equivalent rate in all genotypes (Figure 1a). At the time of inoculation, this tissue had been unopened green buds.

By contrast, there was a significant effect of genotype on silique FAD score $\left(\mathrm{F}_{4,91}=16.23, \mathrm{p}=<0.01\right)$. The siliques assessed had been open flowers at the time of inoculation. The disease progression in the Ler-0 and Ler-0 eds1-2 plants was identical (Figure 1b). The mutant allele $d m r 1-2$ had significantly reduced silique disease levels at all time points compared to eds1-2 (Figure 1b, Figure 2a). This finding was confirmed in multiple independent experiments. Genotypes $d m r 5$ and $d m r 6$ had reduced silique disease symptoms at 7 and 11 days post inoculation (dpi) in the displayed experiment but these findings were not consistent across experiments. The $d m r 1-2$ plants had significantly higher numbers of uninfected green siliques than eds1-2 at all time points, whilst for dmr6 significantly more green siliques were observed at 7 and $11 \mathrm{dpi}$ but not at $14 \mathrm{dpi}$ (Figure 1c).

While healthy rosette leaf tissue is not susceptible to Fusarium infection under our experimental conditions, colonisation by the fungus occurs during leaf senescence. Colonisation of rosette leaves was formally assessed following the observation that $d m r 1$ leaves support less fungal growth. The number of colonised rosette leaves following the initial spray inoculation was found to be significantly affected by genotype $\left(F_{4,93}=66.06, p=<.001\right)$. Both $d m r 1$ alleles had significantly fewer colonised rosette leaves than eds1-2 at all time points (Figure 1d, Figure 2b). Interestingly, Ler-0 had significantly more colonised rosette leaves per plant than eds1-2, indicating that the eds1 mutation may have an effect on F. culmorum leaf susceptibility that was not identified in the previous study which focused on floral infection (Cuzick et al. [11]). 

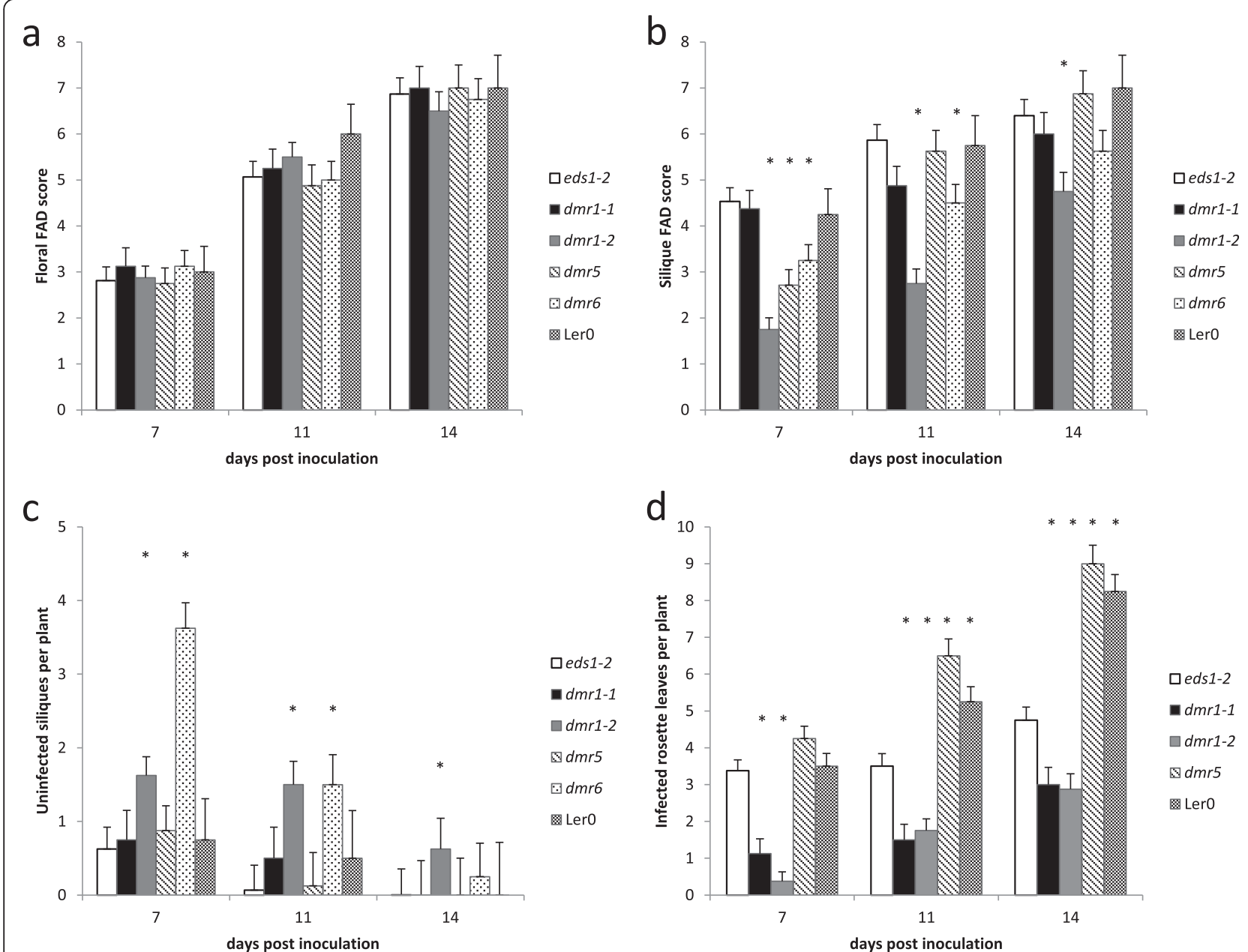

Figure 1 Analysis of susceptibility to F. culmorum infection in four downy mildew resistant (dmr) mutant lines, compared to wild-type Ler-0 and the parental genotype Ler-0 eds 1-2. Six plants per genotype were scored for (a) floral disease levels, (b) silique disease levels, (c) the number of healthy siliques, and (d) the number of infected rosette leaves, at 7, 11 and 14 days post inoculation (dpi). For the floral and silique evaluations the FAD- Fusarium-Arabidopsis Disease scoring system was used. Asterisks indicate genotypes significantly different from eds $1-2$ at each time point (regression analysis followed by calculation of LSDs, $p=<0.05$ ). Error bars represent standard error of the mean. The experiment was repeated with similar results. Since the $d m r 6$ mutant flowers approximately 1 week later than eds $1-2$, dmr6 plants were used in this experiment were 1 week older than those of other genotypes, and therefore rosette leaf data were not comparable due to increased senescence in the dmr6 mutant.

\section{Mutation of DMR1 reduces susceptibility to $F$. graminearum}

FEB disease is caused by several cereal infecting Fusaria species. Therefore, the susceptibility to F. graminearum infection was compared between the dmr1 mutant alleles $d m r 1-1$ and $d m r 1-2$, and the parental genotype eds1-2 at 7, 11 and 14 dpi (Figure 3). Results were similar to those obtained for F. culmorum: No difference was observed in floral susceptibility $\left(\mathrm{F}_{2,62}=2.25, \mathrm{p}=0.114\right)$. Rosette leaf colonisation was affected by genotype $\left(\mathrm{F}_{2}, 62\right.$ $=37.10, \mathrm{p}<.001$ ) with both $d m r 1$ alleles having fewer diseased rosette leaves than eds1-2 (Figure $3 \mathrm{~b}$ and $\mathrm{f}$ ). Silique FAD scores and uninfected silique numbers also differed between genotypes $\left(\mathrm{F}_{2,}, 62=48.63\right.$ and $55.31 \mathrm{re}$ spectively, $\mathrm{p}=<.001)$. Silique FAD scores were lower in dmr1-2 than eds1-2 at all time points, with uninfected green siliques higher in $d m r 1-2$ at 7 and 11 dpi (Figure 3a, $\mathrm{d}$ and e). In these F. graminearum inoculated experiments, full infected and very necrotic siliques were visible in the eds1-2 plants from 7 dpi onwards, whereas this extreme silique phenotype was rarely observed from $11 \mathrm{dpi}$ onwards for either the $d m r 1-1$ or the $d m r 1-2$ plants. Overall these results indicate that both leaf and silique resistance conferred by mutation of DMR1 is conserved across at least two cereal infecting Fusarium species.

\section{Multiple $d m r 1$ alleles have increased resistance to F. culmorum}

In order to verify that the silique resistance phenotype observed in dmr1-2 is a result of mutation of DMR1 and not caused by a second EMS induced mutation, three 


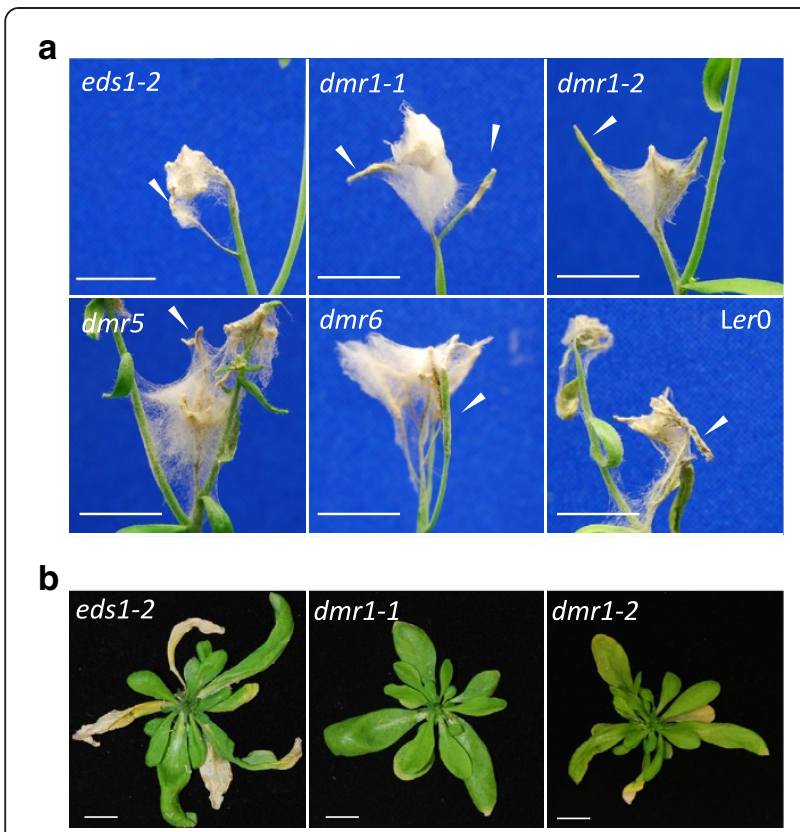

Figure 2 Representative images of the floral and rosette leaf Fusarium culmorum infections of the Arabidopsis downy mildew resistant (dmr) mutants at $\mathbf{1 4} \mathrm{dpi}$, compared to the parental genotype eds 1-2 and wild type Ler-0. (a) Infected floral tissue of all genotypes. (b) Rosette leaves of the $d m r 1$ alleles compared to eds 1-2. The stem and floral tissue has been removed from each plant in panel $\mathrm{b}$. Bar $=1 \mathrm{~cm}$. White arrows = siliques with different levels of infection.

additional alleles of dmr1 (dmr1-3, dmr1-4 and dmr1-6) were tested for altered resistance to F. culmorum (Figure 4). The dmr1-2, dmr1-3, dmr1-4 and dmr1-5 mutants all had lower silique disease levels than eds1-2 (Figure 4a, c) $\left(\mathrm{F}_{5}, 49=2.31, \mathrm{p}=0.005\right)$, whilst no differences in floral susceptibility were observed between the various $d m r 1$ genotypes and eds1-2. This again indicates that the open flowers and very immature siliques at the time of inoculation of the $d m r 1$ mutant plants were more resistant to F. culmorum infection than the green unopened buds. Fungal growth on rosette leaves was also different between genotypes $\left(\mathrm{F}_{5,49}=15.04, \mathrm{p}=<0.001\right)$ with fewer rosette leaves per plant infected in genotypes dmr1-1, dmr1-2, dmr1-3 and dmr1-4 compared to eds1-2 (Figure 4b, d). Collectively, these results confirm that increased silique and leaf resistance occurs in multiple $d m r 1$ alleles and is therefore likely a result of disruption of DMR 1 function.

Plants harbouring the mutant alleles dmr1-1 and dmr1-2 were also assessed for altered susceptibility to $F$. culmorum using a second inoculation method, namely the spore droplet, single silique point inoculation assay. This assay involves initially removing $1 \mathrm{~mm}$ of tissue from the tip of each immature silique and then placing the $1 \mu \mathrm{l}$ spore droplet onto the cut surface. No clear differences were seen in the distance of visible disease progression through the silique and pedicel between genotypes (Additional file 1: Figure S1).

Homoserine accumulates in the siliques of $d m r 1$ mutant plants

Resistance of $d m r 1$ leaves to $H$. arabidopsidis infection was previously linked to elevated homoserine levels in 10 day old seedlings (van Damme et al. [23]). We analysed the amino acid composition of the siliques of three $d m r 1$ mutant alleles compared to eds1-2 in order to identify whether homoserine also accumulates in $d m r 1$ siliques (Figure 5). Homoserine was not detectable in eds1-2 siliques, but was abundant in $d m r 1$ siliques (Figure $5 \mathrm{a}$ ). Homoserine levels were comparable between all three mutant alleles, but were higher on average in $d m r 1-2$ and dmr1-3 siliques, which are resistant to F. culmorum, compared to $d m r 1-1$ siliques, which have wild type resistance levels. As previously observed in seedling tissue by van Damme and colleagues, mutation of homoserine kinase does not reduce levels of downstream amino acids (Figure 5b-e). Threonine levels were elevated in $d m r 1-2$ siliques, while methionine was more abundant in $d m r 1-1$ siliques. Interestingly, these changed levels observed in siliques correlate well with the levels of these amino acids in young seedlings. Glycine (which can be synthesised from threonine) was more abundant in all $d m r 1$ siliques than in eds1-2. An unidentified amino acid that is not a homoserine conjugate was also detected in $d m r 1$ samples but absent from eds1-2.

\section{Exogenous homoserine application reduces $F$. culmorum infection in Arabidopsis buds and siliques}

Exogenous application of L-homoserine, but not Dhomoserine, was previously shown to increase resistance in Arabidopsis and tomato to the obligate biotrophs Hyaloperonospora arabidopsidis, and Oidium neolycopersici, respectively, but homoserine did not inhibit spore germination or in vitro growth of oomycete pathogens (van Damme et al. [23], Huibers et al. [24]). We therefore investigated whether application of either enantiomer of homoserine affected growth of Fusarium in vitro, or had the ability to mimic the $d m r 1$ resistance phenotype in planta. We also investigated the effect of in planta threonine treatment on Fusarium growth, since threonine is elevated in some $d m r 1$ alleles and was previously shown to reduce H. arabidopsidis growth (Stuttmann et al. [25]).

No strong inhibitory effect of either homoserine isoform on in vitro growth was found for F. culmorum or F. graminearum, following $48 \mathrm{~h}$ incubation in synthetic nutrient poor media supplemented with L- or D-homoserine at concentrations ranging from 0 to $80 \mathrm{mM}$ (Additional file 2: Figure S2).

To assess the in planta effects of amino acid treatment on F. culmorum growth, plants were sprayed with either 


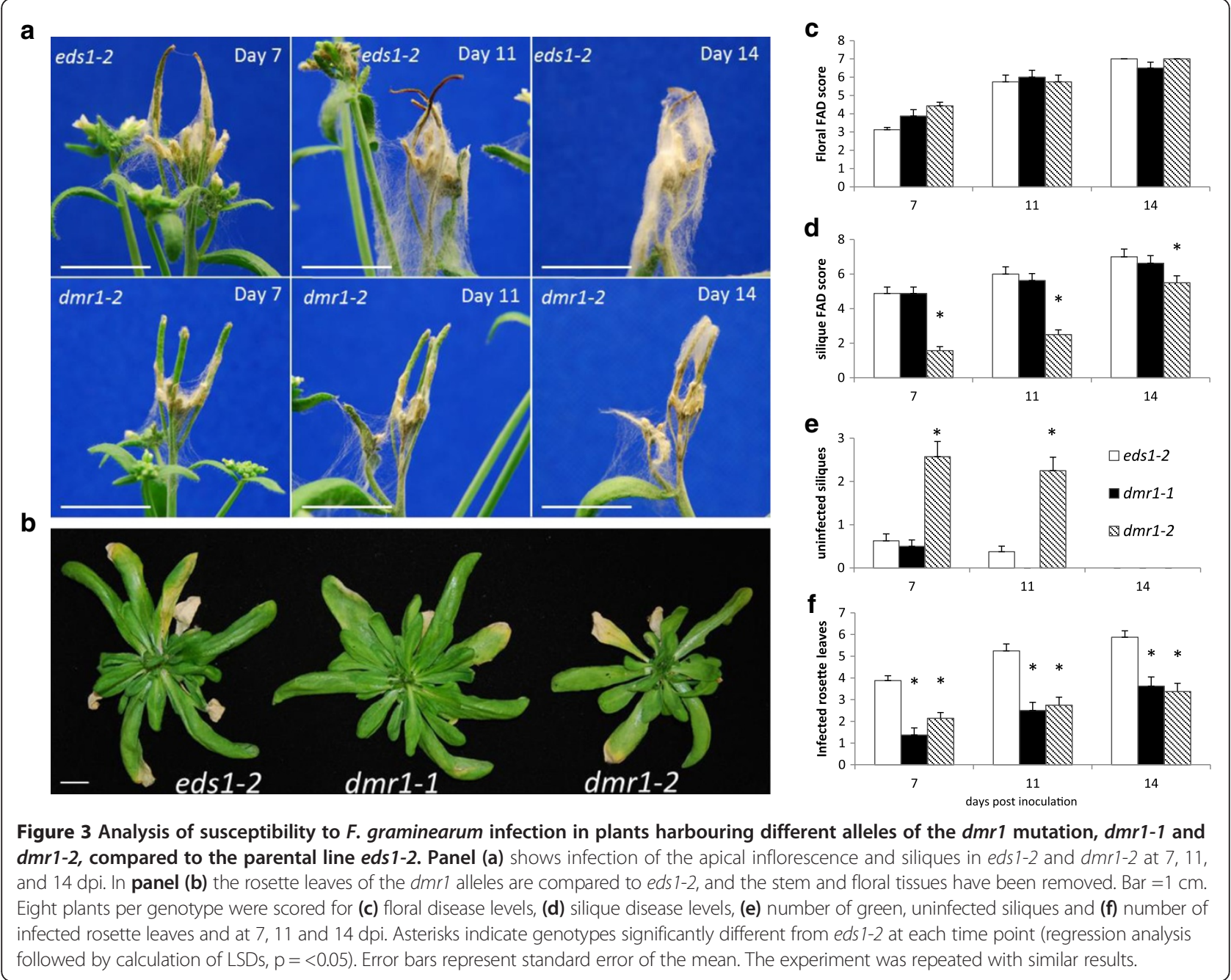

$10 \mathrm{mM} \mathrm{L}$ - or D- homoserine (LHS, DHS), L-threonine (THR) or water, concurrent with spray inoculation with F. culmorum at early flowering. Amino acid/water treatments were repeated daily for $5 \mathrm{dpi}$. Significant differences in F. culmorum infection between treatments were found for unopened buds $\left(\mathrm{F}_{3,31}=41.38, \mathrm{p}=<0.001\right)$, open flowers $\left(F_{3,31}=7.31, p=<0.001\right)$, siliques $\left(F_{3,31}=1.68\right.$, $\mathrm{p}=<0.001)$ and rosette leaves $\left(\mathrm{F}_{3,31}=7.71, \mathrm{p}=<0.001\right)$. At $7 \mathrm{dpi}$, LHS treated buds showed little or no infection, and infection of opened flowers was also reduced, compared to DHS and water treated control plants (Figure 6a, b, c). Silique infection levels were slightly elevated in all amino acid treated plants compared to water controls in these experiments (Figure 6d). Threonine treatment increased F. culmorum colonisation in both open flowers and rosette leaves (Figure 6a and e).

Plants treated with threonine also exhibited leaf chlorosis and lesion formation in the absence of F. culmorum infection, indicating that threonine spray treatment at and above $10 \mathrm{mM}$ may induce a cell death response.
This result was consistent in both eds1-2 and wild type Ler-0 plants (Additional file 3: Figure S3).

We also analysed the effect of D- and L-homoserine on F. culmorum infection of eds1-2 siliques following single silique wound point inoculations (Figure 7). Siliques were droplet inoculated with water, DHS or LHS for 5 days following F. culmorum inoculation. There was a significant difference in F. culmorum infection development between treatments (ANOVA, $\mathrm{p}=<0.001$ ). DHS treatment resulted in a modest reduction in F. culmorum growth along inoculated siliques compared to water treatment, while LHS treatment resulted in significantly less Fusarium growth than either water or DHS treatment, with most plants showing no externally visible infection. However, F. culmorum hyphae were present on and between seeds within some LHS treated siliques with externally uninfected pericarps (Additional file 4: Figure S4).

We also analysed the effect of LHS treatment on $d m r 1$ mutants using the spray treatment method (Figure 8). 


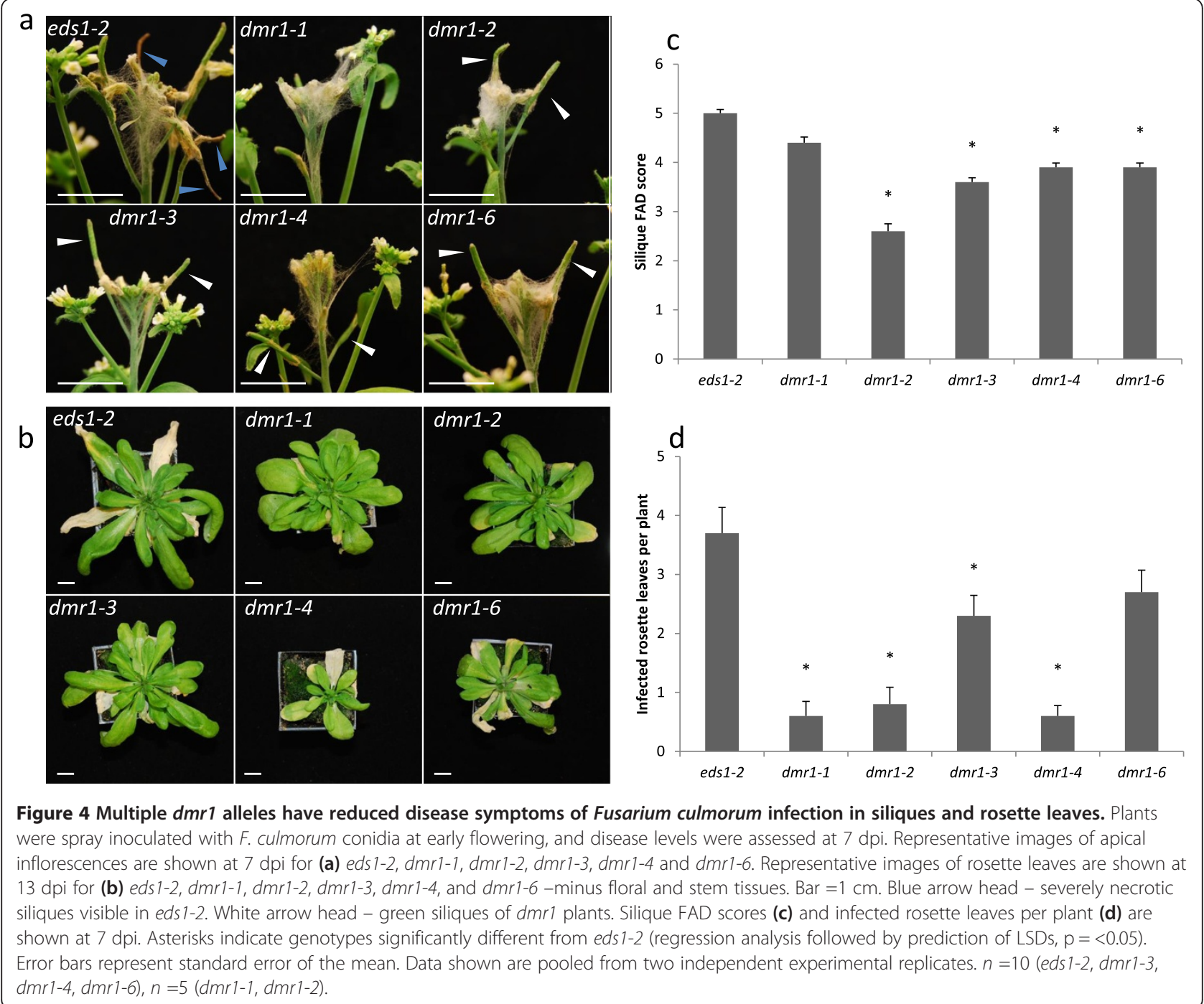

We found that exogenous LHS application, compared to DHS application, conferred F. culmorum resistance in dmr1-2 buds (which are not resistant) equivalent to that seen in LHS treated eds1-2 buds (Figure 6b). Furthermore, LHS treatment afforded a further increase in silique resistance in $d m r 1-2$ siliques, despite a high level of resistance already being conferred by the mutation.

\section{Mutation of DMR1 affects plant growth and senescence}

During the growth of the experimental plants, $d m r 1-2$ plants appeared to be slightly smaller in size than eds1-2 plants. Therefore, the rosette diameter and leaf number were measured and compared between 5-week old plants of genotypes $d m r 1-1, d m r 1-2$ and eds1-2. The quantification of growth confirmed that the $d m r 1-2$ plants have approximately $\sim 25 \%$ smaller rosettes on average than eds $1-2$ (Additional file 5: Figure S5a \& b), but that leaf number is similar between genotypes (Additional file 5: Figure S5c). This supports the recent findings by Huibers et al. [24] that some Arabidopsis $d m r 1$ mutants have reduced fresh weight compared to eds1-2. Leaf senescence between genotypes was also assessed by counting the number of visibly senescent leaves per plant at 7,11 , and 14 days after flowering (corresponding to assessment of infected plants at 7, 11 and $14 \mathrm{dpi}$ ), and found to be delayed in both $d m r 1-1$ and $d m r 1-2$ compared to eds1-2 (Additional file 5: Figure S5d \& e).

Silique number were compared between genotypes at 7, 11 and 14 days post flowering (corresponding to assessment of infected plants at 7, 11 and 14 dpi) to ensure that the increased number of uninfected siliques observed in $d m r 1-2$ was not due to more siliques being produced in this genotype. No difference was found between genotypes at any of the time points assessed (Additional file 5: Figure S5f). There was no evidence of increased silique number in the other $d m r 1$ alleles investigated in this study, although this was not formally assessed. 

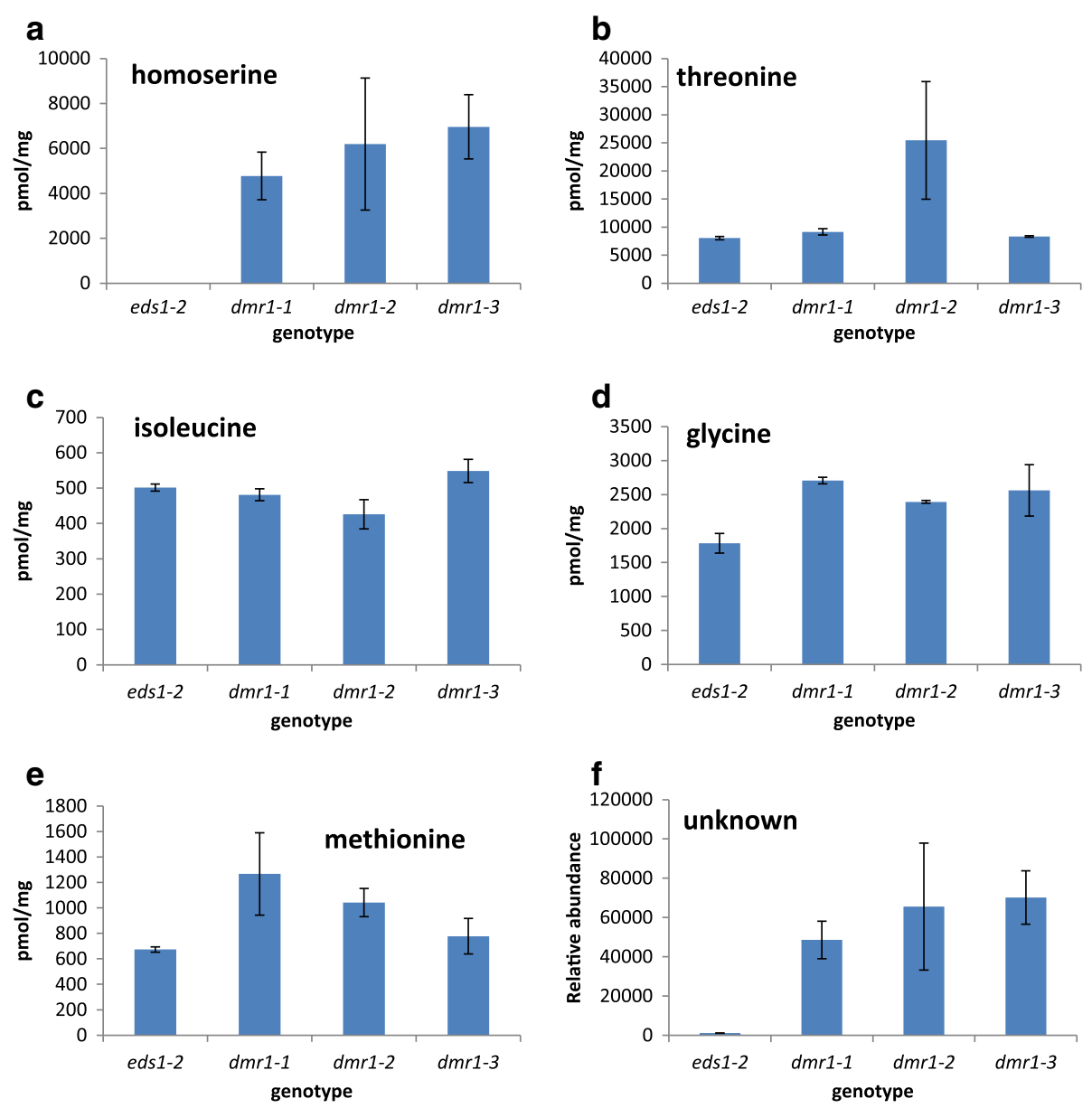

Figure 5 Silique amino acid composition of three $d m r 1$ mutant alleles. Gas chromatography mass spectroscopy (GC-MS) was used to identify and quantify the amino acids present in dmr1-1, dmr1-2 and dmr1-3 compared to eds 1-2 in the absence of Fusarium infection. Homoserine (a) was not detectable in eds 1-2 siliques but was abundant in the siliques of the dmr1 mutants. Despite the absence of a functional homoserine kinase in the dmrl mutants, levels of threonine (b) isoleucine (c) glycine (d) and methionine (e), downstream products of homoserine phosphorylation, were not reduced in the dmr1 mutants compared to eds 1-2. The level of an uncharacterised amino acid (f) was also elevated in all three $\mathrm{dmr} 1$ mutants. Analysis was done on three independent biological silique samples per genotype. Bar =standard error.

Exogenous application of L-homoserine only partially and inconsistently affected Fusarium colonisation of wheat ears The effect of exogenous homoserine application on Fusarium infection in wheat spikes was assessed by treating $F$. graminearum infected wheat spikelets with L-homoserine, D-homoserine or sterile water daily for $7 \mathrm{dpi}$. Fewer mean bleached spikelets and bent awns and higher grain number and weight were observed in L-homoserine treated plants compared to the other treatments in both experimental replicas. However, only the reduced number of bleached spikelets was statistically significant, and only in the first experimental replicate (Additional file 6: Figure S6) $(\mathrm{p}=0.03)$.

\section{Discussion}

In order to identify additional host genes controlling the outcome of the Fusarium-Arabidopsis interaction, as well as highlight components of defence signalling which are conserved in response to different pathogen types, we screened a number of downy mildew resistant mutants for altered susceptibility to the fungal pathogens $F$. culmorum and F. graminearum, which infect floral tissue in cereals and Arabidopsis. We identified that multiple loss of function mutant alleles of the Arabidopsis HOMOSERINE KINASE gene DMR1 have increased resistance to Fusarium culmorum: Siliques of dmr1-2, dmr1-3, dmr1-4 and $d m r 1-6$ were found to be more resistant to infection and leaves of dmr1-1, dmr1-2, dmr1-3 and dmr1-4, supported reduced leaf colonisation. These phenotypes were also observed following F. graminearum infection of $d m r 1-1$ and $d m r 1-2$.

These results indicate that there is potentially a common mechanism of susceptibility occurring in response to infection by both the downy mildew oomycete pathogen 

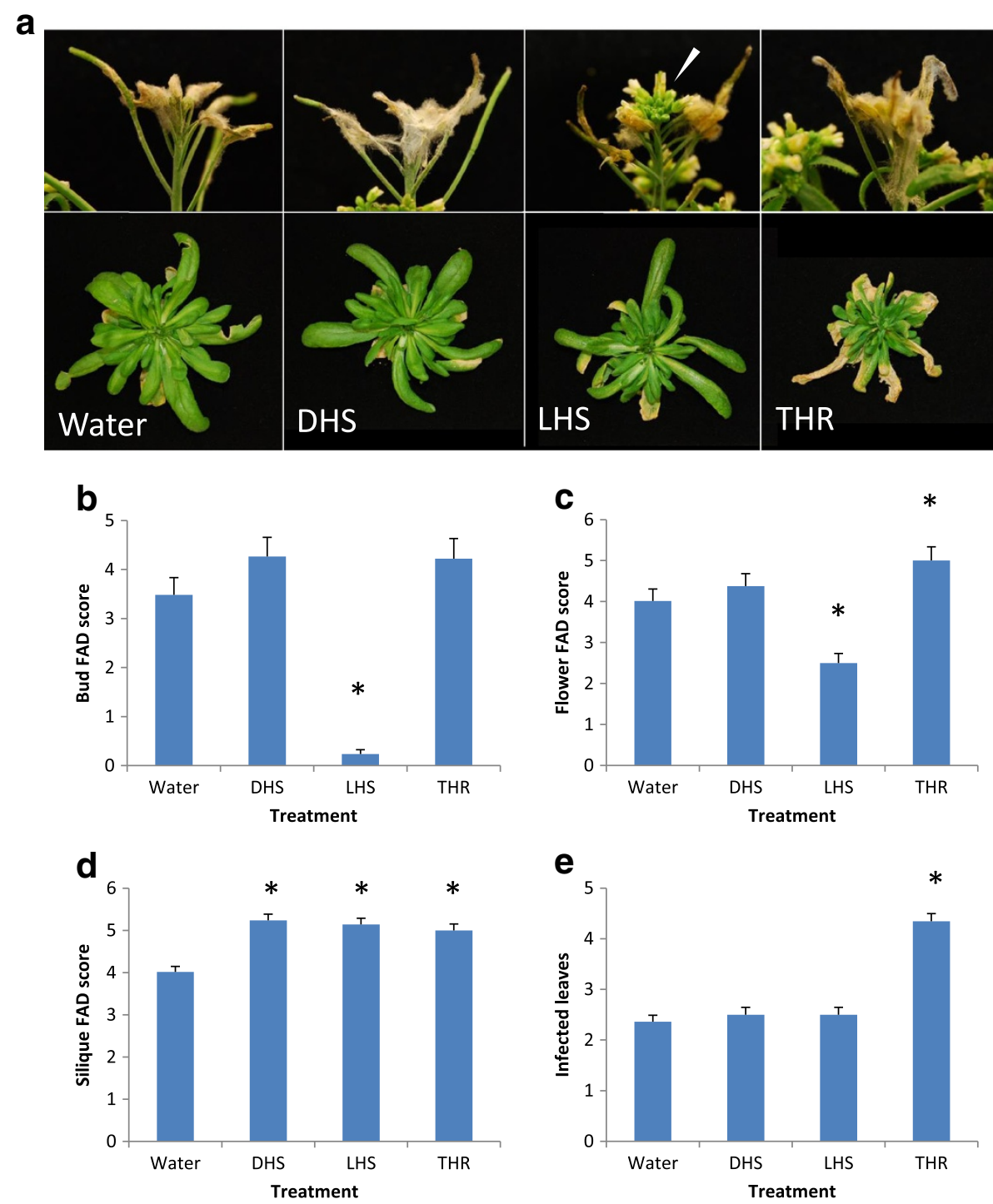

Figure 6 The effect of exogenous amino acid treatments on Fusarium susceptibility in Arabidopsis floral, silique and rosette leaf tissue. Arabidopsis plants of genotype eds 1-2 were sprayed at early flowering with either $10 \mathrm{mM}$ D-homoserine (DHS), L-homoserine (LHS), threonine (THR) or sterile water, co-incident with F. culmorum. Amino acid/water treatments were then repeated daily for $6 \mathrm{dpi}$. Disease was assessed at $7 \mathrm{dpi}$. a) Images show infected apical inflorescences (upper panel) and rosette leaves (lower panel) - stem and floral tissue have been removed from rosettes. White arrow - green and opening buds present in LHS treated plants. Plants were scored for (b) bud disease, (c) open flower disease, (d) silique disease and (e) infected rosette leaf number. Asterisks indicate statistically significant differences from $\mathrm{H}_{2} \mathrm{O}$ treated plants (regression analysis followed by prediction of $L S D s, p=<0.05, n=8$ ). Results are representative of two independent experiments.

H. arabidopsidis, which is a leaf adapted obligate biotroph, and fungal hemi-biotrophic Fusarium species which are floral adapted. Mutation of AtDMR1 and its tomato ortho$\log$ SIDMR1 has also recently been shown to increase resistance to the obligate biotrophic fungal mildew Oidium neolycopersici in an allele dependent manner, but has not been found to alter resistance to any other pathogens assessed, including the facultative biotrophic bacterium Pseudomonas syringae (van Damme et al. [23], Huibers et al. [24]). As with susceptibility to Oidium neolycopersici (Huibers et al. [24]), resistance to Fusarium in dmr1 is allele dependent; while the $d m r 1-1$ allele supports less rosette leaf colonisation and has delayed senescence it does not confer resistance in siliques, despite homoserine accumulating within this tissue. One hypothesis is that homoserine accumulation caused by the $d m r 1$ mutation is not responsible for increased Fusarium silique resistance, and that DMR1 has an additional function contributing to silique susceptibility which is not abolished in the dmr1-1 allele. Meanwhile the delayed leaf senescence and associated reduction in fungal colonisation in this and the other alleles is due to reduced homoserine kinase function, and 


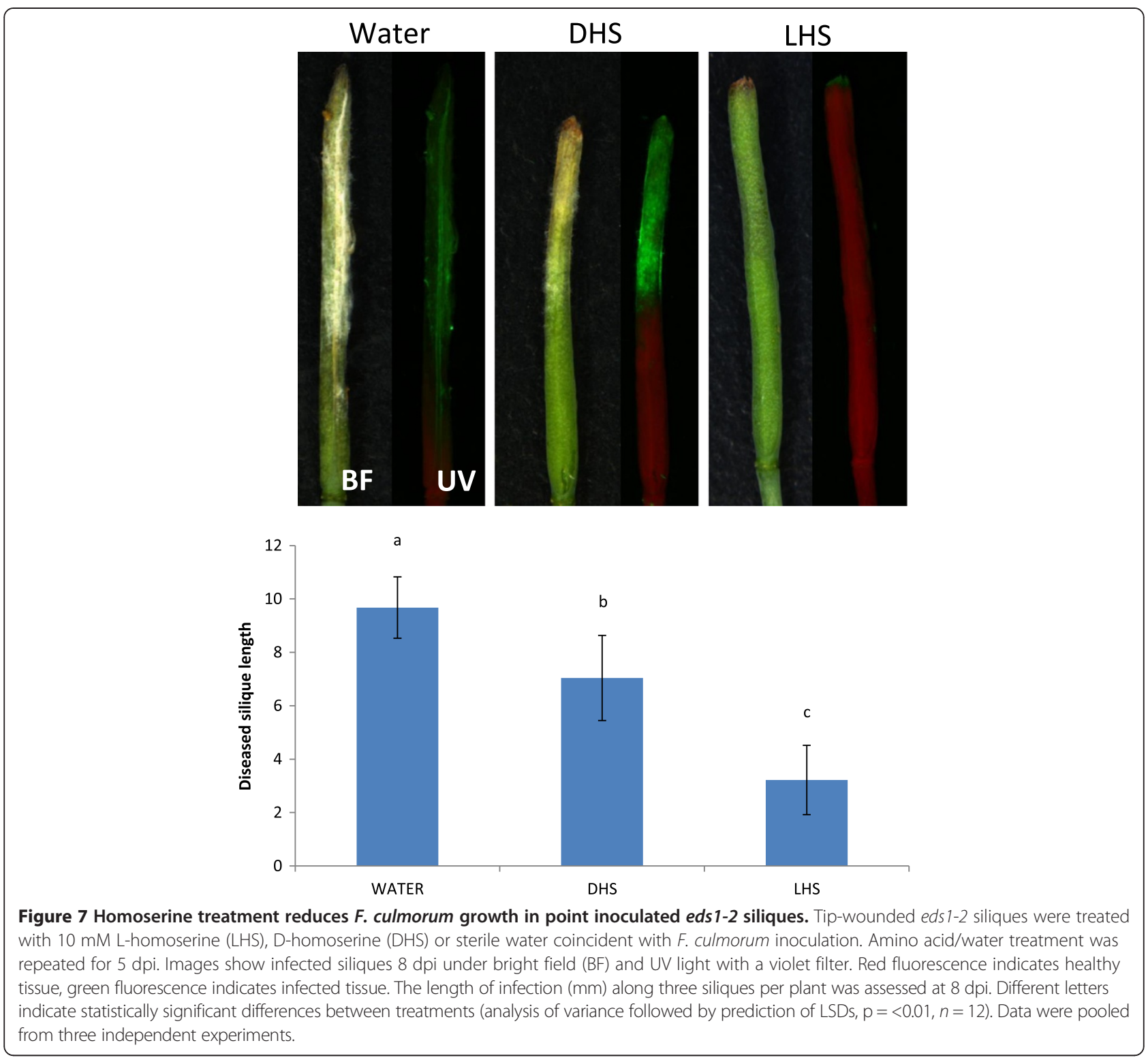

is therefore conserved between alleles. However, this does not account for the reduction in disease afforded by exogenous application of homoserine. An alternative hypothesis is that the dmr1-1 mutant harbours a second mutation as a result of the original EMS mutagenesis which mitigates the silique resistance phenotype, either by blocking the activity of the elevated homoserine or independently elevating silique susceptibility via another pathway. The latter would potentially be less likely to affect saprophytic colonisation of senesced leaf tissue. Further investigation is required to test these hypotheses.

Mutation of DMR1 was not found to alter susceptibility to infection of unopened buds and young flowers, despite affecting both leaf and silique infection. Analysis of Arabidopsis DMR1 expression using Genevestigator (Hruz et al. [26]) shows that DMR1 is expressed at lower levels in some floral tissues than it is in vegetative tissue, namely in the stamens, anthers, stigma and sepals (Additional file 7: Figure S7a). Since homoserine kinase activity has been shown to be driven by homoserine accumulation (Lee et al. [27]), it may be that these tissues do not produce high levels of homoserine and are therefore unaffected by decreased DMR1 activity. Susceptibility of sepal and male reproductive tissue during early floral development pre-fertilisation may result in loss of the flower, whereas infection of these tissues postpollination would have little effect on the siliques of resistant dmr1 plants, as this tissue is shed during silique development.

Since $d m r 1$ induced resistance to $H$. arabidopsidis is proposed to be mediated by homoserine accumulation and can be mimicked by exogenous L-homoserine application 

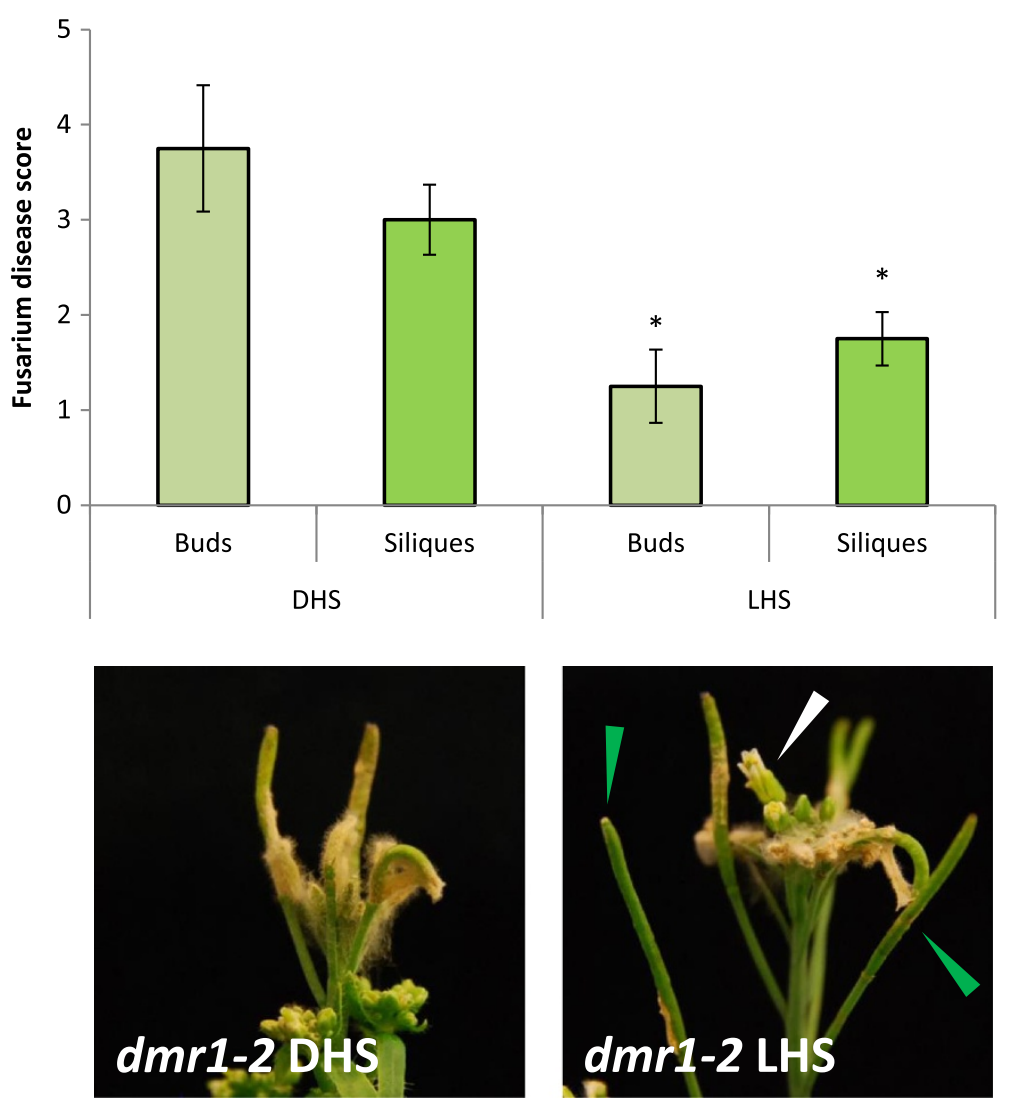

Figure 8 Treatment of $d m r 1-2$ with LHS further reduces Fusarium silique susceptibility compared to DHS treated controls. Arabidopsis plants of genotype dmr1-2 were treated at early flowering with $10 \mathrm{mM} \mathrm{L-homoserine} \mathrm{(LHS)} \mathrm{or} \mathrm{D-homoserine} \mathrm{(DHS)} \mathrm{as} \mathrm{a} \mathrm{control,} \mathrm{concurrent} \mathrm{with}$ F. culmorum spray inoculation. Amino acid/water treatments were repeated for 7 days. Bud and silique disease levels were assessed at $7 \mathrm{dpi}$. Asterisks indicate statistically significant differences between treatments for each tissue type (regression analysis followed by prediction of LSDs, $p=<0.05, n=8$ ). Images show inoculated inflorescences 7 dpi. LHS treatment reduced bud and silique disease levels in $d m r 1-2$. White arrow - opening uninfected buds. Green arrow - green uninfected siliques. Analysis based on pooled data from two independent experiments.

in wild type plants, we investigated the effects of homoserine application on Fusarium growth in vitro and in planta. Treatment of eds1-2 plants with L-homoserine (LHS) following spray inoculation with $F$. culmorum resulted in significantly decreased bud and flower colonisation by the fungus. This contrasts with the phenotype of $d m r 1 \mathrm{mu}-$ tants, which have increased silique and leaf, but not floral, resistance. As previously discussed, the Genevestigator analysis suggested that some floral organs may have lower HSK expression than other plant tissues (Additional file 7: Figure S7). This may result in longer persistence of the applied homoserine in buds than in siliques and other tissues, resulting in reduced fungal growth compared to other tissues. This was corroborated by the finding that exogenous L-homoserine application onto resistant $d m r 1-2$ siliques, which accumulate homoserine, further enhanced resistance to F. culmorum (Figure 8). We did find that more direct application of both the fungus and the LHS onto the tips of wounded siliques resulted in decreased fungal growth along the silique compared to water and DHS treated controls, indicating that homoserine treatment enhances resistance in wild type siliques under these infection conditions. However, in these experiments we also observed less $F$. culmorum infection following treatment with D-homoserine than with water, suggesting that the D-enantiomer of homoserine can also reduce Fusarium growth in certain situations. This finding contrasts with the results obtained following spray inoculation, where both amino acid forms increase silique disease. The biological reason for this is unclear. Formally, it is possible that daily application of distilled water to the silique tip might enhance the growth of Fusarium, which requires high humidity for infection, whereas the various osmotic properties of the amino acid solutions do not. Alternatively the D-homoserine may be eliciting a response from the wounded silique tip which then affects fungal growth. This demonstrates the importance of using the biologically inactive enantiomer as a control.

Exogenous application of homoserine onto the spikelets of $F$. graminearum infected wheat cultivar Apogee did not 
consistently reduce infection. The number of bleached spikelets was significantly lower in LHS treated plants $(\mathrm{p}=0.03)$ at 10 days post inoculation in one experimental replica, but there was no significant difference in the degree of awn bending, grain number or grain weight. Therefore the effect of L-homoserine on Fusarium infection and disease development was less pronounced on wheat floral tissue compared to Arabidopsis. This interspecies difference is likely due to the rapid metabolism of the applied homoserine by the functional wheat homoserine kinase(s), preventing homoserine accumulation and activity. Future research should therefore focus on disruption of wheat homoserine kinase function.

This study also presents the novel finding that exogenous application of threonine induces host cell death in Arabidopsis leaves and increases F. culmorum colonisation. This raises further questions about the effects of amino acid metabolism on plant defence against different pathogen species and lifestyles. van Damme and colleagues did not find an effect of exogenous threonine application on $H$. arabidopsidis susceptibility when amino acids were applied by vacuum infiltration. However, Stuttmann et al. [25] found that spray application of 1-5 mM threonine resulted in decreased $H$. arabidopsidis sporulation in Ler-0 eds1-2 plants. These contrasting outcomes are interesting. $H$. arabidopsidis is a classic obligate biotroph and would therefore be sensitive to any host induced cell death which would limit this pathogen's access to living tissue. By contrast Fusarium has been shown to have a switching in planta lifestyle with host cell death an integral feature of the later disease formation process (Brown et al. [28], Desmond et al. [29], Thaler et al. [30]). Cereal infecting Fusaria are also able to colonise saprophytically dead plant tissue. Threonine mediated chlorosis may therefore facilitate Fusarium colonisation while preventing growth of obligate biotrophic pathogens.

Related to this is the finding that mutation of $D M R 1$ results in delayed senescence. Analysis of DMR1 expression during plant development using GENEVESTIGATOR (Hruz et al. [26]) shows that expression is fairly static throughout plant development but increases during senescence (Additional file 7: Figure S7b). This suggests that DMR1 function could have a role in programmed cell death and senescence. The delayed DMR1 dependent cell death in the $d m r 1$ mutants may restrict Fusarium disease progression and prevent its successful exploitation of host cell death (Thaler et al. [30]). For pathogens such as Fusarium with a combined hemi-biotrophic and saprophytic life style strategy, delayed cell death could prevent full tissue exploitation and the gaining of additional nutrition from the cellular debris. This is supported by the finding that $d m r 1$ leaves display delayed senescence accompanied by a decrease in Fusarium colonisation. However, the delayed cell death may not be the underlying cause of the enhanced resistance. For example, the manner in which delayed cell death might help protect plants against obligate biotrophic pathogens such as $H$. arabidopsidis is not clear. It is formally possible that the normal amino acid ratios found in healthy Arabidopsis tissue are modified in the $d m r 1$ mutants and this alters the efficiency of nutrient acquisition via the haustoria interfaces in obligate biotrophic interactions as well as altering the switching lifestyle of hemibiotrophic pathogens. In this regard, the identification of the novel accumulating amino acid in the three $d m r$ mutants, but not eds1-2 (Figure 5) remains a priority.

Huibers et al. [24] found a correlation between reduced Arabidopsis plant fresh weight in different $d m r 1$ mutant alleles and the level of resistance conferred to O. neolycopersici. The authors concluded that it might be difficult to obtain $d m r 1$ alleles in crop species which conferred enhanced resistance to this pathogen in the absence of a fitness cost. While the dmr1-3 mutant allele did not confer a significant growth penalty, likewise it did not confer resistance to $O$. neolycopersici. However, in the current study, dmr1-3 conferred resistance to F. culmorum in both the leaf and silique tissue of Arabidopsis, although the leaf phenotype was not as strong as in other alleles. Investigation into the effects of homoserine kinase disruption in FEB-susceptible cereal crops is therefore warranted. This may be achieved both through stable transgenesis and the use of an inducible promoter to drive an RNAi construct. Alternatively, the use of transient Virus Induced Gene Silencing (Lee et al. [31], Lee et al. [32]) of cereal homoserine kinases could be deployed.

In this study and that of van Damme et al. [21] it was found that $d m r 1$ plants have wild type or elevated levels of the amino acids methionine, threonine and isoleucine, in both foliar and silique tissue (Figure 5). These amino acids are understood to be synthesised directly via the activity of homoserine kinase. The abundance of these three amino acids in plants with severely reduced homoserine kinase function therefore challenges the current understanding of amino acid biosynthetic pathways. It may be the case that these amino acids are being synthesised via alternative, currently unidentified pathway(s) in the $d m r 1$ mutants, which are independent of homoserine kinase. Alternatively, mutated homoserine kinase may retain some residual function: Homoserine is synthesised via the activity of Arabidopsis aspartate kinases, which are negatively regulated by accumulation of S-adenosylmethionine (SAM), synthesised from methionine (Curien et al. $[33,34])$. Reduced methionine biosynthesis may therefore result in increased aspartate kinase activity, shunting more homoserine into the pathway. Some of the accumulating homoserine might then be phosphorylated by the mutated homoserine kinase, restoring equilibrium in the pathway. However, no changes were observed in 
aspartate levels between wild type and $d m r 1$ mutant plants. Experiments to compare aspartate kinase expression and activity between genotypes would be informative.

\section{Conclusions}

This study has identified that a series of mutations in the Arabidopsis homoserine kinase gene DMR1 confers resistance in silique tissue to the primary causal agents of cereal FEB disease, a source of crop yield losses and grain contamination. Siliques of the $d m r 1$ mutants accumulate homoserine, and exogenous application of Lhomoserine confers resistance to the floral and silique tissues of both mutant $d m r 1$ and wild type DMR1 plants. These finding offer the possibility of developing a novel source of resistance to an economically important floral crop disease for which few other resistance mechanisms exist. Further work will use virus induced gene silencing of the wheat DMR1 ortholog to explore the potential of homoserine in Fusarium resistance in wheat. However, the mechanism by which homoserine accumulation in plant tissue mediates resistance is still not fully understood, and may be key to fully exploiting $d m r 1$ based resistance which has the potential for use in multiple crop species.

\section{Methods}

\section{Plant growth}

The Arabidopsis downy mildew resistant mutant collection and parent genotype eds1-2 were a gift from Guido van den Ackerveken, Utrecht University, The Netherlands. Ecotype Ler-0 (NASC stock NW20) was a gift from Graham McGrann, John Innes Centre, UK. Plants were grown in Levingtons F2 + S compost in a Fitotron 'walk in' plant growth chamber (www.fitotron.co.uk), with a $16 \mathrm{~h}$ light/ $8 \mathrm{~h}$ dark cycle at temperatures of $20^{\circ} \mathrm{C}$ (light) and $17^{\circ} \mathrm{C}$ (dark), with $150 \mu \mathrm{mol} \mathrm{m}{ }^{-2} \mathrm{~s}^{-1}$ fluorescent illumination, at $70 \%$ humidity.

\section{Fusarium growth and storage}

F. culmorum strain 98/11 (NRRL 54112) and F. graminearum strain PH-1 (NRRL 31084) were propagated as previously described (Urban et al. [9]; Cuzick et al. [10]); Conidia were transferred from frozen stocks stored at $-80^{\circ} \mathrm{C}$ onto synthetic nutrient poor agar plates (SNA $-0.1 \% \mathrm{KH}_{2} \mathrm{PO}_{4}, 0.1 \% \mathrm{KNO}_{3}, 0.1 \% \mathrm{MgSO}_{4} \times 7 \mathrm{H}_{2} \mathrm{O}, 0.05 \%$ $\mathrm{KCl}, 0.02 \%$ glucose, $0.02 \%$ saccharose, $2 \%$ agar) for $8-11$ days, and then transferred onto potato dextrose agar (PDA) plates for $48 \mathrm{~h}$ to encourage high levels of conidial production. Conidia were then suspended in sterile distilled water and filtered through sterile Miracloth $\left(\right.$ Calbiochem $\left.^{\oplus}\right)$, and stored at $-80^{\circ} \mathrm{C}$ prior to plant inoculations. Studies using F. graminearum strain PH-1 were conducted under PHSI license 101948/198285/2.

\section{Arabidopsis-Fusarium infection assays}

For spray inoculation assays, plants were selected at early flowering (2-4 open flowers per plant) and the whole plant spray inoculated with $F$. culmorum or F. graminearum conidia suspended in sterile water at a concentration of $10^{6}$ conidia/ml. Control plants were spray inoculated with sterile water.

For amino acid treatment studies, inoculated plants were sprayed daily with a solution of $10 \mathrm{mM}$ L-homoserine, D-homoserine or L-threonine, or sterile water as a control. This was repeated daily for 5 days post inoculation.

For single silique wound point inoculations, plants with six young siliques were selected for inoculation. The top $1 \mathrm{~mm}$ of each silique was removed using sterile scissors and the tip inoculated with a $1 \mu$ l droplet containing approx. $10^{5}$ Fusarium conidia suspended in sterile $\mathrm{H}_{2} \mathrm{O}$. Sterile $\mathrm{H}_{2} \mathrm{O}$ alone was used as a control. For amino acid treatments, conidial suspensions were supplemented at the time of inoculation with $20 \mathrm{mM}$ L-homoserine or D-homoserine, or sterile water as a control. Amino acid suspensions were then re-applied for 6 days post inoculation.

Plants were transferred to inoculation boxes at $100 \%$ relative humidity for the duration of the experiment and kept in darkness for $20 \mathrm{~h}$ following inoculation.

\section{Scoring of Fusarium disease symptoms}

Plants were scored for floral and silique disease symptoms using the Fusarium-Arabidopsis disease (FAD) scoring system devised by Urban et al. [9]. Disease scores per plant were assigned from 0 (no disease symptoms) to 7 (severe disease symptoms with constriction of the main stem) for apical flowers and siliques (Additional file 8: Table S1). The silique score applies to the most diseased silique on each plant, only increasing if all siliques display equivalent disease, and does not therefore take account of the number of siliques escaping disease. For this reason, uninfected green siliques per plant were also counted. The number of diseased rosette leaves per plant was also counted. At least five plants per treatment or genotype were used in each experiment, and each experiment was done at least twice.

\section{In vitro Fusarium growth tests}

F. culmorum and F. graminearum conidia at a concentration of $2 \times 10^{5}$ conida $\mathrm{ml}^{-1}$ were cultured for two days in 96 well flat bottomed culture plates in $200 \mu \mathrm{l}$ synthetic nutrient poor liquid media supplemented with either L- or Dhomoserine at a concentration range from 0 to $80 \mathrm{mM}$. Absorbance as a surrogate for fungal growth was measured for each homoserine concentration as previously described (Fan et al. [35]). Three biological replicates were included per fungal isolate/amino acid treatment, and the experiment repeated. 


\section{Wheat infection and amino acid treatment}

The dwarf wheat cultivar Apogee (Bugbee, Koerner et al. [36]) was used for wheat infection assays. The 8th and 9th spikelets of ears at anthesis were point inoculated with $5 \mu \mathrm{l}$ of F. graminearum conidial suspension at $10^{5} \mathrm{ml}^{-1}$. In addition, the 6th-11th spikelets were treated with $5 \mu \mathrm{l}$ of either $10 \mathrm{mM}$ L-homoserine or D-homoserine, or sterile water. The three different treatments were then repeated daily for 7 days. The number of bleached spikelets and bent awns (preceding bleaching in infected spikelets) was assessed, and grain weight and number recorded at $10 \mathrm{dpi}$ following dissection of the rachis, as per Baldwin et al. [37].

\section{Analysis of silique amino acids}

The amino acid content of $15 \mathrm{mg}$ freeze dried and ground silique samples from $d m r 1$ mutant and eds1-2 plants was analysed using the EZFaast GC-MS physiological amino acid analysis kit, according to the manufacturers' instructions. The protocol was amended such that the addition of the internal standard supplied with the kit was omitted and the final solvent evaporation step with reconstitution in organic solvent was replaced with a 1:10 dilution with the organic solvent (reagent 6). Samples were analysed on an Agilent 5975 Inert MSD coupled to a 7890A Gas Chromatograph fitted with a Zebron Amino acid ZB-AAA column $(10 \mathrm{~m} \times 0.25 \mathrm{~mm}$ I.D. Phenomenex, Cheshire, UK), Gestel MPS2 autosampler and split/splitless injector (fitted with quartz wool packed SGE FocusLiner). For each genotype three biological replicates were analysed, each consisting of siliques from $\sim 8$ pooled 6 -week old plants. The internal standard, amino acid standard solutions and glutamine standard were obtained from Sigma (Dorset, UK). Homoserine standard was obtained from Koch-Light Laboratories, Colnworth, Bucks, UK.

\section{Microscopy}

Fusarium infected Arabidopsis siliques were imaged using a Leica M205 FA stereomicroscope and accompanying LAS-AF6000 software, using white light or UV light with a Violet filter (Excitation: 425/40 nm, Emission: $475 \mathrm{~nm}$ ).

\section{Statistical analysis}

The Arabidopsis - Fusarium disease susceptibility data were subjected to regression analysis fitted to a general linear model with assumed Poisson distribution. For mutant experiments with multiple time points, the effects of genotype and time, and the interactions between genotype and time, were examined. Where a significant effect of genotype or treatment was found ( $\mathrm{p}=<0.05)$, genotype/ treatment means and least significant differences (LSDs) between genotypes or treatments were calculated at a 5\% confidence level and means for all genotypes compared to genotype eds1-2, and all amino acid treatments compared to water treatment, to identify significant differences. In the absence of an interaction between genotype and time, one mean per genotype was predicted by amalgamating data from all time points, with corresponding LSDs. Where a significant interaction between genotype and time was observed, means and LSDs to eds1-2 were calculated for each genotype at each time point assessed. For analysis of the effect of LHS on dmr1-2 compared to eds1-2 LSDs between each genotype/treatment combination were used to identify statistically significant differences between treatments. For single silique point wound inoculations, analysis of variance (ANOVA) was used to compare fungal growth (in $\mathrm{mm}$ ) along the silique. LSDs ( $p=0.01)$ were calculated between treatments. All statistical analysis was done using Genstat v16 (Payne et al. [38]).

\section{Supporting data}

All relevant supporting data can be found within the supplementary files accompanying to this article.

\section{Arabidopsis mutant loci}

The following Arabidopsis loci are associated with this study: dmr1; AT2G17265, dmr6; AT5G24530, eds1-2; AT3G48090. Further information can be obtained from www.arabidopsis.org.

\section{Additional files}

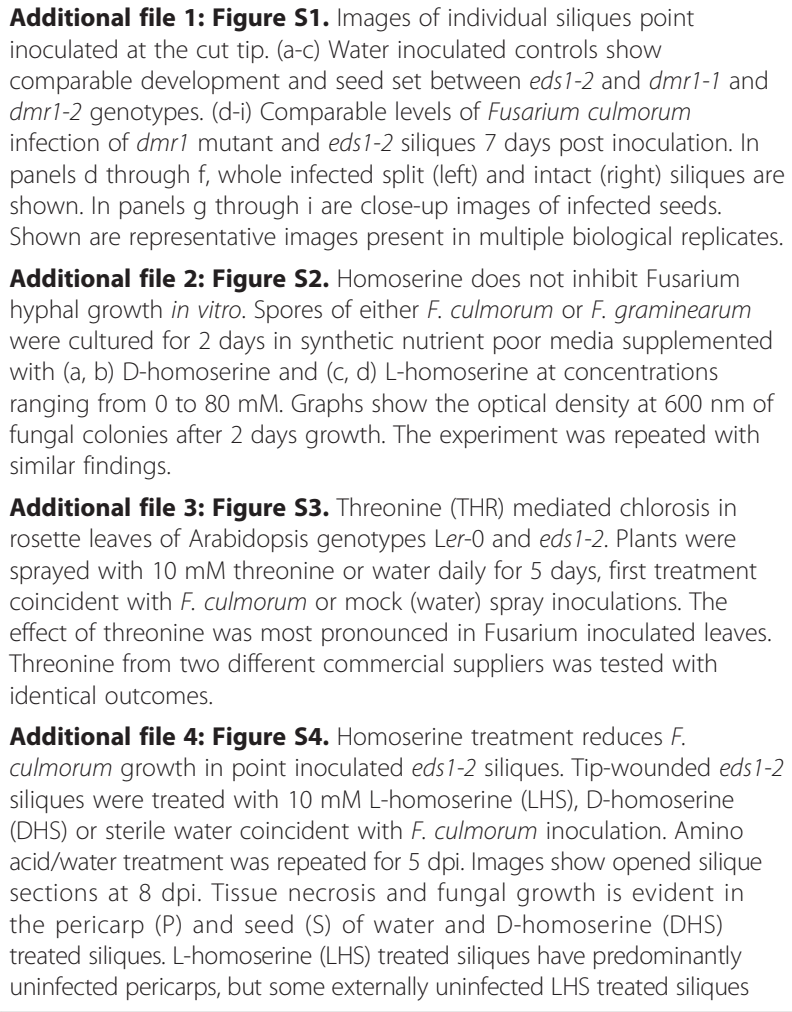

Additional file 3: Figure S3. Threonine (THR) mediated chlorosis in rosette leaves of Arabidopsis genotypes Ler-0 and eds 1-2. Plants were sprayed with $10 \mathrm{mM}$ threonine or water daily for 5 days, first treatment coincident with F. culmorum or mock (water) spray inoculations. The effect of threonine was most pronounced in Fusarium inoculated leaves. Threonine from two different commercial suppliers was tested with identical outcomes.

Additional file 4: Figure S4. Homoserine treatment reduces $F$. culmorum growth in point inoculated eds $1-2$ siliques. Tip-wounded eds $1-2$ siliques were treated with $10 \mathrm{mM} \mathrm{L-homoserine} \mathrm{(LHS),} \mathrm{D-homoserine}$ (DHS) or sterile water coincident with F. culmorum inoculation. Amino $\mathrm{acid} /$ water treatment was repeated for $5 \mathrm{dpi}$. Images show opened silique sections at $8 \mathrm{dpi}$. Tissue necrosis and fungal growth is evident in the pericarp (P) and seed (S) of water and D-homoserine (DHS) treated siliques. L-homoserine (LHS) treated siliques have predominantly uninfected pericarps, but some externally uninfected LHS treated siliques 
revealed, when opened, the presence of fungal colonisation within the silique (far right).

Additional file 5: Figure S5. Differences in developmental morphology and senescence between the dmr1-1 and dmr1-2 mutant alleles and eds 7-2. (a, b) Rosette diameter is reduced in 5-week old plants of genotype dmr1-2 compared to eds 1-2. (c) Leaf number is comparable between genotypes. $(d, e)$ Leaf senescence is delayed in both dmr1-1 and dmr1-2. Panel $d$ shows the appearance of the rosettes of flowering plants at 14 days post flowering. (f) Silique number was equivalent between all genotypes throughout seed set. These phenotypes were observed across multiple experimental replicates. Asterisks indicate significant difference from eds $1-2 .{ }^{*} p<0.05,{ }^{* *} p=<0.01$ ( $b-$ ANOVA, $e-$ Regression analysis).

Additional file 6: Figure S6. Effect of $\mathrm{L}$-homoserine application on Fusarium infection of wheat. Spikes of wheat cultivar Apogee were point inoculated with $F$. graminearum and then treated with either L-homoserine (LHS) D-homoserine (DHS) or water for 7 days. The number of bent awns (a) and bleached spikelets (b) along with grain weight (c) and number (d) per plant were assessed at $10 \mathrm{dpi}$. (*) The number of bleached spikelets was significantly lower in LHS treated plants $(p=0.03)$. No statistically significant difference between treatments was found for other parameters (ANOVA, $p=>0.05)$. Bar $=$ standard error

Additional file 7: Figure S7. GENEVESTIGATOR analysis of the expression profile of Arabidopsis DMR1. A) Tissue specific expression levels across different floral tissues. B) Development stage specific expression levels.

Additional file 8: Table S1. Scoring of Fusarium disease in Arabidopsis floral and silique tissue, adapted from Urban et al. [9]. Plants were given separate scores for floral and silique infection from 0 (no disease) to 7 (constriction of the main stem). The intermediate scores of 2 and 4 (F), and 2, 4 and 6 (S) were reserved for when all the tissue on a single plant exhibited the disease phenotype described for the preceding score.

\section{Competing interests}

The authors declare that they have no competing interests.

\section{Authors' contributions}

HB designed and carried out all experiments and data analysis, with the exception of GC-MS. NH carried out GC-MS and subsequent data analysis. KHK participated in experimental design, manuscript drafting and editing and supervision of the research project. All authors read and approved the final manuscript.

\section{Acknowledgements}

The authors would like to thank Julian Franklin and colleagues for maintenance of controlled environment facilities, Stephen Powers for assistance with statistical analysis and Guido van den Ackerveken for providing Arabidopsis seeds. Microscopy was done in the Bioimaging facility at Rothamsted Research. We are grateful to Drs Jason Rudd and Martin Urban for their comments on the manuscript and to Professor Peter Hedden for discussion on this research.

This work was done as part of a PhD Quota studentship (S2055) funded by the BBSRC (Biotechnology and Biological Sciences Research Council, UK). Rothamsted Research receives grant-aided support from the BBSRC. KHK receives additional support from within the BBSRC Institute Strategy Grant 20:20 wheat $(B B / J / 00426 \mathrm{X} / 1)$

Received: 24 July 2014 Accepted: 6 November 2014

Published online: 29 November 2014

\section{References}

1. Dean R, Van Kan JA, Pretorius ZA, Hammond-Kosack KE, Di Pietro A, Spanu PD, Rudd JJ, Dickman M, Kahmann R, Ellis J, Foster GD: The top 10 fungal pathogens in molecular plant pathology. Mol Plant Pathol 2012, 13:414-430.

2. Goswami RS, Kistler HC: Heading for disaster: Fusarium graminearum on cereal crops. Mol Plant Pathol 2004, 5:515-525.

3. Parry DW, Jenkinson P, Mcleod L: Fusarium ear blight (Scab) in small-grain cereals - a review. Plant Pathol 1995, 44:207-238.
4. Rocha O, Ansari K, Doohan FM: Effects of trichothecene mycotoxins on eukaryotic cells: a review. Food Addit Contam 2005, 22:369-378.

5. Bai GH, Shaner G: Management and resistance in wheat and barley to Fusarium head blight. Annu Rev Phytopathol 2004, 42:135-161.

6. Buerstmayr $\mathrm{H}, \mathrm{Ban} \mathrm{T}$, Anderson JA: QTL mapping and marker-assisted selection for Fusarium head blight resistance in wheat: a review. Plant Breed 2009, 128:1-26.

7. Jayatilake DV, Bai GH, Dong YH: A novel quantitative trait locus for Fusarium head blight resistance in chromosome 7A of wheat. Theor Appl Genet 2011, 122:1189-1198.

8. Zhou MP, Hayden MJ, Zhang ZY, Lu WZ, Ma HX: Saturation and mapping of a major Fusarium head blight resistance QTL on chromosome 3BS of Sumai 3 wheat. J Appl Genet 2010, 51:19-25.

9. Urban M, Daniels S, Mott E, Hammond-Kosack K: Arabidopsis is susceptible to the cereal ear blight fungal pathogens Fusarium graminearum and Fusarium culmorum. Plant J 2002, 32:961-973.

10. Cuzick A, Lee S, Gezan S, Hammond-Kosack KE: NPR1 and EDS11 contribute to host resistance against Fusarium culmorum in Arabidopsis buds and flowers. Mol Plant Pathol 2008, 9:697-704.

11. Cuzick A, Maguire K, Hammond-Kosack KE: Lack of the plant signalling component SGT1b enhances disease resistance to Fusarium culmorum in Arabidopsis buds and flowers. New Phytol 2009, 181:901-912.

12. Makandar R, Essig JS, Schapaugh MA, Trick HN, Shah J: Genetically engineered resistance to Fusarium head blight in wheat by expression of Arabidopsis NPR1. Mol Plant Microbe Interact 2006, 19:123-129.

13. Makandar R, Nalam V, Chaturvedi R, Jeannotte R, Sparks AA, Shah J: Involvement of salicylate and jasmonate signaling pathways in Arabidopsis interaction with Fusarium graminearum. Mol Plant Microbe Interact 2010, 23:861-870.

14. Savitch L, Subramaniam R, Allard G, Singh J: The GLK1 'regulon' encodes disease defense related proteins and confers resistance to Fusarium graminearum in Arabidopsis. Biochem Biophys Res Commun 2007, 359:234-238.

15. Van Hemelrijck W, Wouters PFW, Brouwer M, Windelinckx A, Goderis IJWM De Bolle MFC, Thomma BPHJ, Cammue BPA, Delauré SL: The Arabidopsis defense response mutant esa 1 as a model to discover novel resistance traits against Fusarium diseases. Plant Sci 2006, 171:585-595.

16. Asano T, Miwa A, Maeda K, Kimura M, Nishiuchi T: The secreted antifungal protein thionin 2.4 in Arabidopsis thaliana suppresses the toxicity of a fungal fruit body lectin from Fusarium graminearum. PLoS Pathog 2013, 9:8

17. Ferrari S, Sella L, Janni M, De Lorenzo G, Favaron F, D'Ovidio R: Transgenic expression of polygalacturonase-inhibiting proteins in Arabidopsis and wheat increases resistance to the flower pathogen Fusarium graminearum. Plant Biol 2012, 14(1):31-38.

18. Kaur J, Thokala M, Robert-Seilaniantz A, Zhao P, Peyret H, Berg H, Pandey S, Jones J, Shah D: Subcellular targeting of an evolutionarily conserved plant defensin MtDef4.2 determines the outcome of plant-pathogen interaction in transgenic Arabidopsis. Mol Plant Pathol 2012, 13(9):1032-1046.

19. Koch A, Kumar N, Weber L, Keller H, Imani J, Kogel K-H: Host-induced gene silencing of cytochrome P450 lanosterol C14a-demethylase-encoding genes confers strong resistance to Fusarium species. Proc Nat Acad SCi 2013, 110(48):19324-19329.

20. Schreiber KJ, Nasmith CG, Allard G, Singh J, Subramaniam R, Desveaux D: Found in translation: high-throughput chemical screening in Arabidopsis thaliana identifies small molecules that reduce Fusarium head blight disease in wheat. Mol Plant Microbe Interact 2011, 24:640-648.

21. Van Damme M, Andel A, Huibers RP, Panstruga R, Weisbeek PJ, Van den Ackerveken G: Identification of Arabidopsis loci required for susceptibility to the downy mildew pathogen Hyaloperonospora parasitica. Mol Plant Microbe Interact 2005, 18:583-592.

22. Van Damme M, Huibers RP, Elberse J, Van den Ackerveken G: Arabidopsis DMR6 encodes a putative 20G-Fe(II) oxygenase that is defense-associated but required for susceptibility to downy mildew. Plant J 2008, 54:785-793.

23. Van Damme M, Zeilmaker T, Elberse J, Andel A, De Sain-Van Der Velden M, Van Den Ackerveken G: Downy mildew resistance in Arabidopsis by mutation of HOMOSERINE KINASE. Plant Cell 2009, 21:2179-2189.

24. Huibers RP, Loonen AE, Gao D, Van den Ackerveken G, Visser RG, Bai Y: Powdery mildew resistance in tomato by impairment of SIPMR4 and SIDMR1. PLoS One 2013, 8:e67467. 
25. Stuttmann J, Hubberten HM, Rietz S, Kaur J, Muskett P, Guerois R, Bednarek $P$, Hoefgen R, Parker JE: Perturbation of Arabidopsis amino acid metabolism causes incompatibility with the adapted biotrophic pathogen Hyaloperonospora arabidopsidis. Plant Cell 2011, 23:2788-2803.

26. Hruz T, Laule O, Szabo G, Wessendorp F, Bleuler S, Oertle L, Widmayer P, Gruissem W, Zimmermann P: Genevestigator V3: a reference expression database for the meta-analysis of transcriptomes. Adv Bioinformatics 2008, 2008:5. Article ID 420747

27. Lee M, Martin MN, Hudson AO, Lee J, Muhitch MJ, Leustek T: Methionine and threonine synthesis are limited by homoserine availability and not the activity of homoserine kinase in Arabidopsis thaliana. Plant J 2005, 41:685-696.

28. Brown NA, Urban M, van de Meene AM, Hammond-Kosack KE: The infection biology of Fusarium graminearum: defining the pathways of spikelet to spikelet colonisation in wheat ears. Fungal Biol 2010, 114:555-571.

29. Desmond OJ, Manners JM, Stephens AE, Maclean DJ, Schenk PM, Gardiner DM, Munn AL, Kazan K: The Fusarium mycotoxin deoxynivalenol elicits hydrogen peroxide production, programmed cell death and defence responses in wheat. Mol Plant Pathol 2008, 9:435-445.

30. Thaler JS, Owen B, Higgins VJ: The role of the jasmonate response in plant susceptibility to diverse pathogens with a range of lifestyles. Plant Physiol 2004, 135:530-538.

31. Lee WS, Rudd JJ, Hammond-Kosack KE, Kanyuka K: Mycosphaerella graminicola LysM effector-mediated stealth pathogenesis subverts recognition through both CERK1 and CEBiP homologues in wheat. Mol Plant Microbe Interact 2013, 27(3):236-243.

32. Lee WS, Hammond-Kosack KE, Kanyuka K: Barley stripe mosaic virus-mediated tools for investigating gene function in cereal plants and their pathogens: virus-induced gene silencing, host-mediated gene silencing, and virus-mediated overexpression of heterologous protein. Plant Physiol 2012, 160:582-590.

33. Curien G, Laurencin M, Robert-Genthon M, Dumas R: Allosteric monofunctional aspartate kinases from Arabidopsis. FEBS J 2007, 274(1):164-176.

34. Curien G, Ravanel S, Robert M, Dumas R: Identification of six novel allosteric effectors of Arabidopsis thaliana aspartate kinase-homoserine dehydrogenase isoforms: physiological context sets the specificity. J Biol Chem 2005, 280(50):41178-41183.

35. Fan J, Urban M, Parker JE, Brewer HC, Kelly SL, Hammond-Kosack KE, Fraaije BA, Liu X, Cools HJ: Characterization of the sterol 14a-demethylases of Fusarium graminearum identifies a novel genus-specific CYP51 function. New Phytol 2013, 198:821-835

36. Bugbee B, Koerner G, Albrechtsen R, Dewey W, Clawson S: Registration of cultivars: registration of 'USU-Apogee' wheat. Crop Sci 1997, 37(2):626.

37. Baldwin TK, Gaffoor I, Antoniw J, Andries C, Guenther J, Urban M, Hallen-Adams HE, Pitkin J, Hammond-Kosack KE, Trail F: A partial chromosomal deletion caused by random plasmid integration resulted in a reduced virulence phenotype in Fusarium graminearum. Mol Plant Microbe Interact 2010, 23(8):1083-1096.

38. Payne RW, Murray DA, Harding SA, Baird DB, Soutar DM: An Introduction to GenStat for Windows. 14th edition. Hemel Hempstead, UK: VSN International 2011.

doi:10.1186/s12870-014-0317-0

Cite this article as: Brewer et al: Mutations in the Arabidopsis

homoserine kinase gene DMR1 confer enhanced resistance to

Fusarium culmorum and F. graminearum. BMC Plant Biology

2014 14:317

\section{Submit your next manuscript to BioMed Central and take full advantage of:}

- Convenient online submission

- Thorough peer review

- No space constraints or color figure charges

- Immediate publication on acceptance

- Inclusion in PubMed, CAS, Scopus and Google Scholar

- Research which is freely available for redistribution 